\title{
Lentivirus-delivered nemo-like kinase small interfering RNA inhibits laryngeal cancer cell proliferation in vitro
}

\author{
JUN TAI $^{1,2}$, YUANSHENG RAO ${ }^{3}$, JUGAO FANG $^{4,5}$, ZHIGANG HUANG $^{4,5}$, ZHENKUN YU $^{4,5}$, \\ XIAOHONG CHEN ${ }^{4,5}$, WEIGUO ZHOU ${ }^{4,5}$, XIAO XIAO $^{1,2}$, TING LONG $^{1,2}$, \\ YANG HAN ${ }^{1,2}$, QIAOYIN LIU ${ }^{1,5}$, AIDONG LI ${ }^{6}$ and $\mathrm{XIN} \mathrm{NI}^{2,4}$ \\ ${ }^{1}$ Beijing Key Laboratory for Pediatric Diseases of Otolaryngology, Head and Neck Surgery, \\ Beijing Pediatric Research Institute, Beijing Children's Hospital, Capital Medical University; \\ ${ }^{2}$ Department of Otolaryngology Head and Neck Surgery, Beijing Children's Hospital, Capital Medical University, \\ Beijing 100045; ${ }^{3}$ Department of Otolaryngology Head and Neck Surgery, Beijing Anzhen Hospital, Capital Medical University, \\ Beijing 100029; ${ }^{4}$ Key Laboratory of Otolaryngology-Head and Neck Surgery, Ministry of Education, Beijing Tongren Hospital, \\ Capital Medical University, Beijing 100005; ${ }^{5}$ Department of Otolaryngology Head and Neck Surgery, \\ Beijing Tongren Hospital, Capital Medical University, Beijing 100730; ${ }^{6}$ Department of Center Laboratory, \\ Cancer Institute and Hospital, Chinese Academy of Medical Sciences, Beijing 100021, P.R. China
}

Received March 5, 2014; Accepted December 3, 2014

DOI: $10.3892 / \mathrm{mmr} .2015 .4189$

\begin{abstract}
Laryngeal squamous cell carcinoma is the most common form of head and neck squamous cell carcinoma. Multiple approaches have been applied to treat this type of cancer; however, no significant improvement in survival rate has been achieved. In the present study, the role of nemo-like kinase (NLK) in human laryngeal carcinoma Hep-2 cells was investigated. NLK has been identified as an important regulator of cell growth, patterning and cell death in a variety of organisms. Lentivirus-mediated-shRNA was employed to silence endogenous NLK expression. Downregulation of the expression of NLK following lentivirus infection was confirmed using reverse transcription quantitative polymerase chain reaction and western blot analysis. The effects of NLK downregulation on Hep-2 cell proliferation and cell cycle progression were analyzed using an MTT assay and flow cytometry, respectively. Downregulation of NLK also inhibited tumorigenesis and regulated the expression of cell cycle protein expression levels. Therefore, it was hypothesized
\end{abstract}

Correspondence to: Dr Aidong Li, Department of Center Laboratory, Cancer Institute and Hospital, Chinese Academy of Medical Sciences, 17 Panjiayuan Nanli, Chaoyang, Beijing 100021, P.R. China

E-mail: aidonglidr@126.com

Dr Xin Ni, Department of Otolaryngology Head and Neck Surgery, Beijing Children's Hospital, Capital Medical University, 56 Nanlishi Lu, Xicheng, Beijing 100045, P.R. China

E-mail: nixinnixin@126.com

Key words: nemo-like kinase, Hep-2 cells, lentivirus, short hairpin RNA, proliferation, tumorigenesis that NLK is necessary for cell survival and tumorigenesis in laryngeal cancer cells. Furthermore, the absence of NLK may lead to cancer cell death. Collectively, the results of the present study demonstrated that the lentivirus-mediated targeted disruption of NLK may be a promising therapeutic method for the treatment of laryngeal cancer.

\section{Introduction}

Head and neck squamous cell carcinoma is the sixth most common type of cancer, with laryngeal squamous cell carcinoma becoming more common with an increasing occurrence of new cases and mortality annually $(1,2)$. The highest incidence rates in males are registered in Asia at 12 cases per 100,000 individuals per year (3). Laryngeal cancer patients usually lose their laryngeal function, which affects speech, swallowing and breathing; thus, normal activities of the patients are impaired (4). At present, surgery and radiotherapy are the predominant treatments and are often followed by chemotherapy, particularly in locally advanced disease (5). Multiple approaches have been applied to treat this type of cancer; however, the survival rate of patients has not significantly improved. Therefore, laryngeal carcinoma represents a target for developing novel therapeutic approaches. An improved understanding of the mechanisms involved in the regulation of cell proliferation requires the identification of novel genes associated with laryngeal carcinoma. Additionally, the upregulation or downregulation of these genes may be important in the treatment of this severe disease.

Nemo-like kinase (NLK) has been identified as an important regulator of cell growth, patterning and cell death in a variety of organisms $(6,7,8)$. NLK was originally isolated as a murine orthologue of the Drosophila Nemo gene by reverse transcription polymerase chain reaction 
(RT-PCR) from neural tissue mRNA of the embryonic mouse using degenerate primers designed for the conserved kinase domains I, VI, VII and IX of the extracellular-signal regulated kinase/mitogen-activated protein kinase (MAPK) family. NLK, an evolutionarily conserved serine/threonine kinase, belongs to the proline directed protein kinase superfamily, consisting of MAPK and cyclin-dependent protein kinases (CDKs) (9). Genetic studies of the homologs of NLK in animal models have demonstrated that NLK has a suppressive role in Wnt/ $\beta$-catenin signaling $(10,11)$.

RNA silencing, also known as RNA interference (RNAi) is a form of post-transcriptional gene silencing mediated by a short double-stranded small interfering RNA (siRNA). This method has been demonstrated to be highly effective for gene knockdown and holds great promise in the field of cancer therapy (12). A number of gene products involved in carcinogenesis have already been examined as targets for RNAi and RNAi-targeting of molecules crucial for tumor-host interactions and tumor resistance to chemo or radiotherapy has also been investigated. Long-lasting RNAi-based gene silencing can be achieved with the aid of lentivirus-based expression systems, which drive the production of short hairpin RNA (shRNA) species. Lentiviral vectors are an appealing tool for transgenesis in part due to their ability to incorporate into genomic DNA with high efficiency. In addition, they can also be maintained for long periods of time (13). Lentiviral vectors have become a promising tool for the establishment of transgenic animals and the manipulation of the mammalian genome.

\section{Materials and methods}

Cell culture. Hep-2 cells were obtained from the CEll Bank of the Chinese Academy of Sciences (Shanghai, China) maintained in Dulbecco's modified Eagle's medium (DMEM; HyClone, GE Healthcare, Little Chalfont, UK) containing 10\% fetal bovine serum (Biowest, Loire Valley, France), 1\% l-glutamine and $1 \%(\mathrm{v} / \mathrm{v})$ penicillin-streptomycin (Sigma-Aldrich, St. Louis, MO, USA). The cells were subcultured following trypsinization once or twice per week in a 1:5 split ratio. The cell lines were maintained as monolayers in $75 \mathrm{~cm}^{2}$ cell culture flasks at $37^{\circ} \mathrm{C}$ in a humidified atmosphere of $5 \% \mathrm{CO}_{2}$.

Lentivirus infection. Cells were incubated with lentivirus in a small volume of serum-free DMEM at $37^{\circ} \mathrm{C}$ for $4 \mathrm{~h}$. Subsequently, 10\% DMEM was added and the cells were placed in an incubator for 4-5 days for the following experiments. Lentivirus was used to infect Hep-2 cells at a multiplicity of infection (MOI) of 10 , and the infection efficiency was evaluated by green fluorescent protein (GFP) on the premise that no virus toxic effect was observed. Thus, the following experiments were performed using viruses at an MOI of 10, unless otherwise indicated. The lentivirus packaged with GFP was used for the cell proliferation assays and the lentivirus without GFP was used for the cell apoptosis propidium iodide (PI) staining to exclude interference with the GFP signal. The Hep-2 cells transfected with the NLK-siRNA (5'-GATAGACCTATTGGATATG-3') and the negative control siRNA (5'-TTCTCCGAACGTGTCACGT-3') were designated Lv-shNLK and Lv-shCon, respectively. Furthermore, to determine the infection efficiency, cells expressing GFP protein were observed using fluorescence microscopy (CKX41; Olympus, Tokyo, Japan) 5 days after infection.

Quantitative polymerase chain reaction ( $q P C R)$. At 5 days after infection, total RNA was extracted from control, Lv-shNLK and Lv-shCon using TRIzol reagent (Invitrogen Life Technologies, Carlsbad, CA, USA). The following primer sequences were used for the detection of NLK: NLK, sense 5'-ATCATCAGCACTCGCATCATC-3' and antisense 5'-GACCAGACAACACCAAAGGC-3'; GAPDH, sense 5'-TGACTTCAACAGCGACACCCA-3' and antisense 5'-CACCCTGTTGCTGTAGCCAAA-3'. GAPDH was used as an internal control. The PCR mixture was prepared using SYBR Green master mix (Takara Bio Inc., Ohtsu, Japan) in accordance with the manufacturer's instructions. All RT-qPCR experiments were performed using an Mx3000P thermal cycler (Agilent Technologies, Santa Clara, CA, USA).

Western blot analysis. At 5 days after infection, the proteins from Hep-2 cells were analyzed using western blot analysis. Standard procedures were used for western blotting. Briefly, Hep-2 cells were washed with ice-cold phosphate-buffered saline (PBS; Sigma-Aldrich), scraped and then lysed in radioimmunoprecipitation assay buffer. Cell debris was removed by centrifugation at $10,300 \mathrm{x} \mathrm{g}$ for five minutes followed by flash freezing of the supernatants. The protein concentrations were determined according to the bicinchoninic acid assay (BCA Protein Assay reagent, 23227; Pierce, Thermo Fisher Scientific, Rockford, IL, USA). Total protein (30 $\mu \mathrm{g})$ was subjected to a $10 \%$ SDS-PAGE and electroblotted onto a polyvinylidene difluoride membrane (EMD Millipore, Billerica, MA, USA). The membranes were blocked with 5\% bovine serum albumin for $2 \mathrm{~h}$ and subsequently incubated with different antibodies, which were used to detect respective proteins using an electrochemiluminescence assay kit (Pierce) according to the manufacturer's instructions. The antibodies used were as follows: Rabbit polyclonal anti-NLK (1:500; ab116715; Abcam, Cambridge, UK); mouse monoclonal anti-Cyclin B1 (1:1,000; cat. no. K0128-3; Medical \& Biological Laboratories, Aichi, Japan); mouse monoclonal anti-Cyclin D1 (1:1,000; cat. no. MD-17-3; Medical \& Biological Laboratories); rabbit polyclonal anti-P53 (1:1,000; cat. no. 9282; Cell Signaling Technology, Inc., Danvers, MA, USA); rabbit polyclonal anti-Phospho-p53 (Ser315) (1:1000; cat. no. 2528; Cell Signaling Technology, Inc.); rabbit polyclonal anti-GAPDH (1:60,000; cat. no. 10494-1-AP; Proteintech Group, Inc, Chicago, IL, USA). The secondary antibodies were goat anti-rabbit immunoglobulin G (IgG) (1:5,000; cat. no. SC-2054; Santa Cruz Biotechnology, Inc., Dallas, TX, USA) and goat anti-mouse IgG (1:5,000; cat. no. SC-2005; Santa Cruz Biotechnology, Inc.).

Cell viability assay. Cells were cultured in 96-well culture dishes at a density of $1 \times 10^{4}$ cells/well. Following infection for 1, 2, 3, 4 and 5 days, the media in each well were removed and the cell viability value was measured using an MTT colorimetric assay. To determine the cell viability, $20 \mu \mathrm{l}$ MTT $(5 \mathrm{mg} / \mathrm{ml}$ ) was added to each well and cells were incubated for an additional $4 \mathrm{~h}$. The supernatant was then removed and the 
A

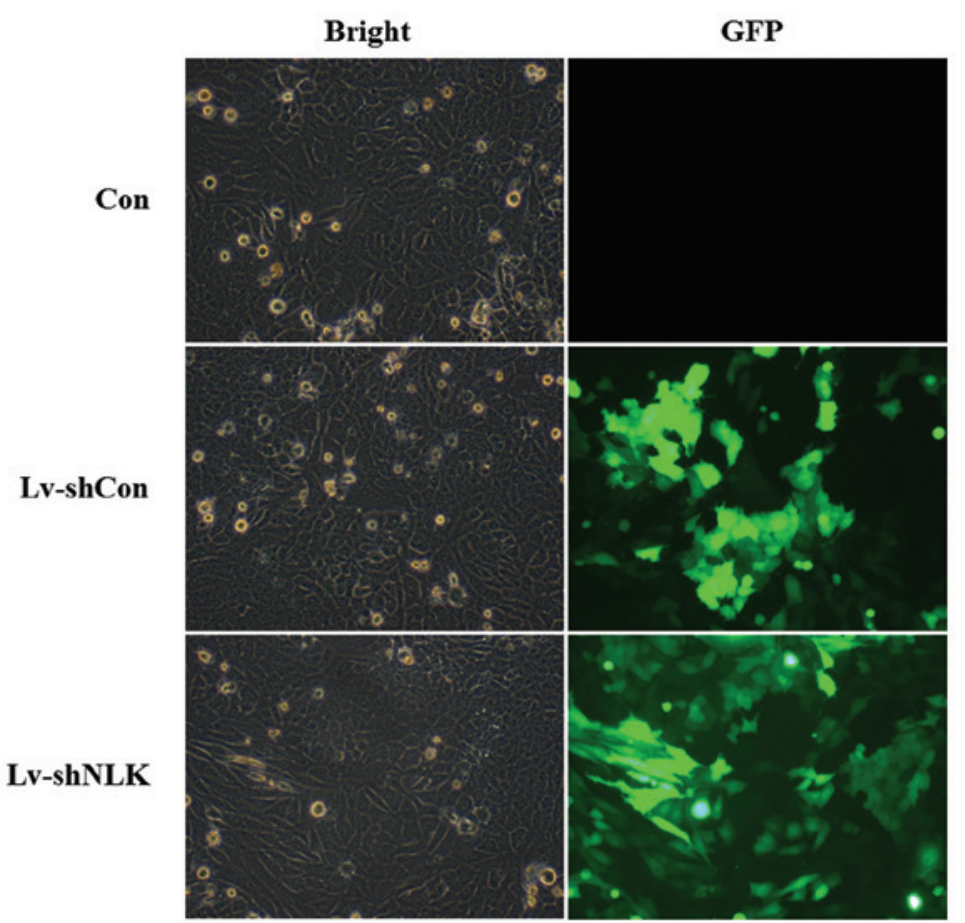

B

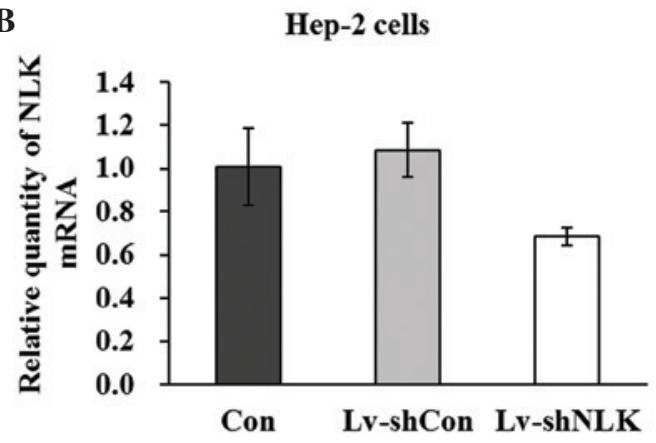

C

\section{NLK}

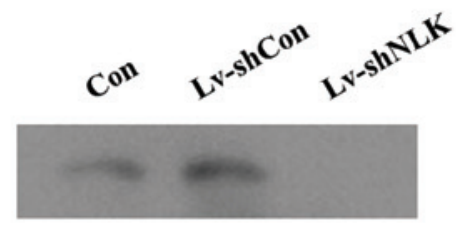

GAPDH

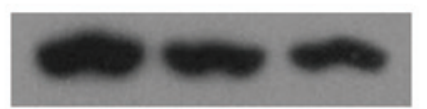

Figure 1. Lentiviral vectors for the NLK siRNA were constructed and demonstrated to be specific and potent for silencing NLK expression in Hep-2 cells. (A) Expression of GFP from uninfected Hep-2 cells (Con) and cells infected with the Lv-shNLK and Lv-shCon indicates the lentivirus infection efficiency in Hep-2 cells (magnification, x10). (B) Quantitative polymerase chain reaction of NLK mRNA levels from uninfected Hep-2 cells (Con) and cells infected with the Lv-shNLK and Lv-shCon. (C) Western blot analysis of NLK protein expression in uninfected Hep-2 cells (Con) and cells infected with the Lv-shNLK and Lv-shCon. NLK, nemo-like kinase; GFP, green-fluorescent protein.

insoluble formazan product was dissolved in acidified isopropanol. The optical density (OD) of the 96-well culture plates was measured using an enzyme-linked immunosorbent assay reader (2550; BioTek Instruments, Inc., Winooski, VT, USA) at $595 \mathrm{~nm}$. The OD of formazan formed in untreated control cells was interpreted as $100 \%$ viability.

Cell cycle analysis by flow cytometry. At each indicated time point, the treated cells were trypsinized and pooled with the floating cells in the culture medium. The cells were collected by centrifugation at $300 \mathrm{x}$ g for $5 \mathrm{~min}$. The cell pellet was mildly resuspended in a solution containing $584 \mu \mathrm{g} / \mathrm{ml} \mathrm{NaCl}$, $1,000 \mu \mathrm{g} / \mathrm{ml}$ sodium citrate, $10 \mu \mathrm{g} / \mathrm{ml}$ RNAase A, $0.3 \mu \mathrm{g} / \mathrm{ml}$ Nonidet P-40 and $50 \mu \mathrm{g} / \mathrm{ml}$ PI. The cell suspensions were incubated for $30 \mathrm{~min}$ in the dark at room temperature, followed by the addition of a solution containing $15 \mathrm{mg} / \mathrm{ml}$ citric acid, $0.25 \mathrm{mM}$ sucrose and $50 \mu \mathrm{g} / \mathrm{ml}$ PI. The suspension of PI-stained isolated nuclei were mixed and kept in the dark at $4^{\circ} \mathrm{C}$ prior to flow cytometric measurement. The cell cycle distributions were analyzed on a FACScan (Becton-Dickinson, Franklin Lakes, NJ, USA).

Colony formation assay. Briefly, $0.5 \mathrm{ml}$ base layers consisting of $0.8 \%$ agar medium were prepared in six-well plates Uninfected and infected cells were trypsinized, centrifuged at $105 \mathrm{x} \mathrm{g}$ for $5 \mathrm{~min}$, resuspended in $0.4 \%$ agar medium (equal volumes of $0.8 \%$ noble agar and culture medium) and plated onto the top agar at 200 cells/well. The cells were cultured for 14 days at $37^{\circ} \mathrm{C}$. Following culturing for 14 days, adherent cells were washed twice with PBS and then fixed with $4 \%$ paraformaldehyde for $30 \mathrm{~min}$ at room temperature. Colonies were visualized using a cell staining Giemsa solution (Chemicon, Temecula, CA, USA) and counted under a fluorescence microscope.

Statistical analysis. The results of all assays are expressed as the mean \pm standard deviation. All assays were performed independently in triplicate. Statistical analyses were performed using Student's t-test. $\mathrm{P}<0.05$ was considered to indicate a statistically significant difference.

\section{Results}

Expression of NLK in Hep-2 cells is suppressed by infection with si-NLK. In order to knock down the NLK gene in Hep-2 cells, GFP containing lentivirus-mediated infection of NLK siRNA was performed. The successful infection was confirmed by evaluating the expression level of GFP. As shown in Fig. 1A, 5 days after NLK infection, $>90 \%$ of cells expressed GFP, indicating a high infection efficiency. The mRNA expression level of NLK following infection with siRNA was evaluated by qPCR. Quantitative analysis of NLK mRNA level using densitometry revealed a significant decrease in Lv-shNLK (36.6\%) compared with the control (Fig. 1B). In addition, western blot assays demonstrated that the protein expression of NLK in the two Hep-2 cell lines was significantly suppressed by NLK-specific siRNA expression (Fig. 1C). Collectively, these data suggested that lentivirus-mediated siRNA successfully infected Hep-2 cells and in addition, may be used to investigate NLK loss of function effects in Hep-2 cells. 
A

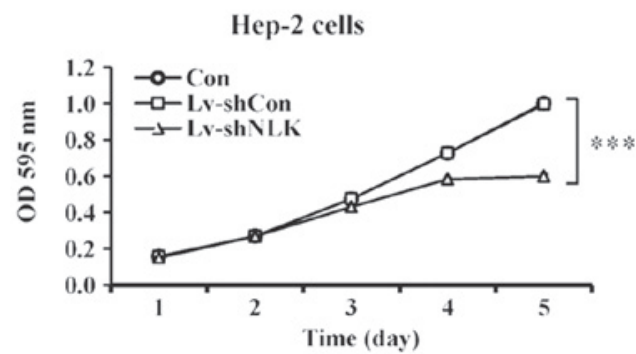

C

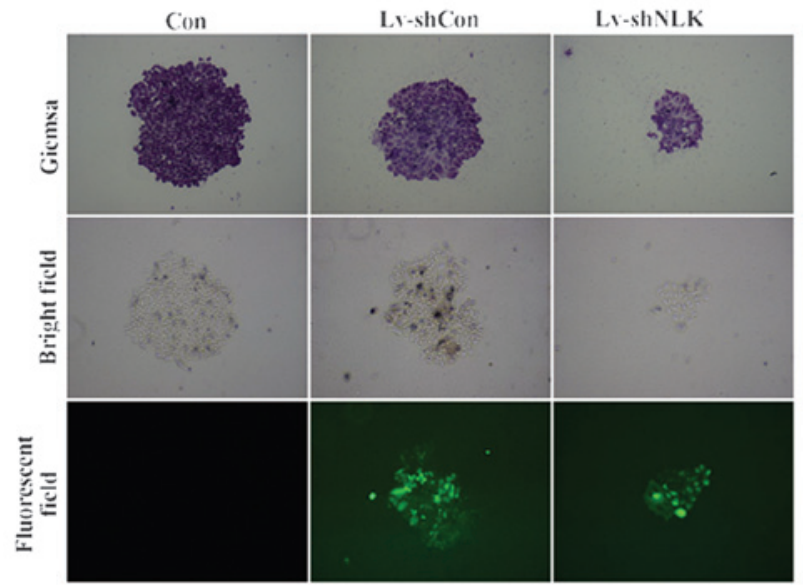

B

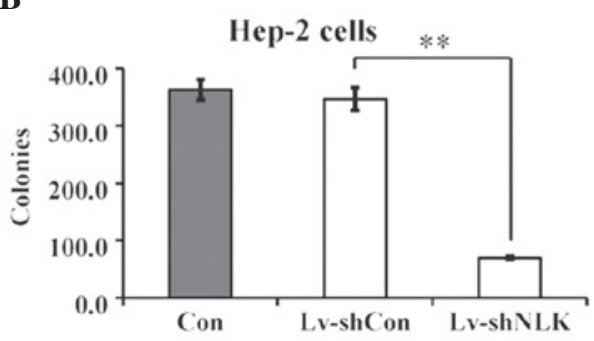

D

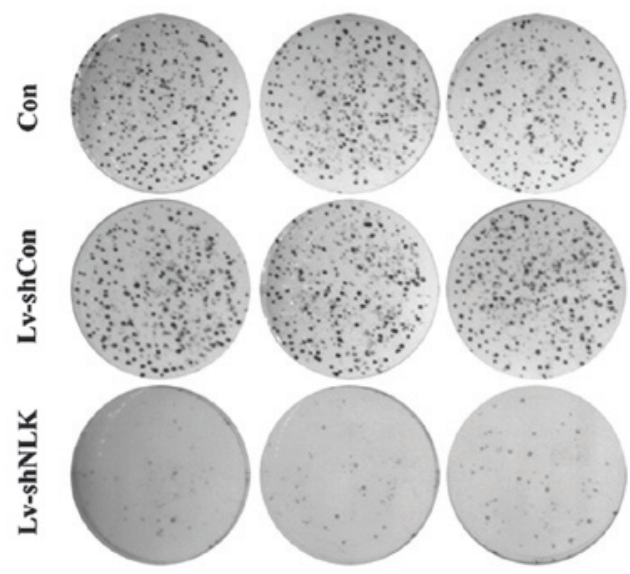

Figure 2. NLK regulates the cell proliferation and colony formation of Hep-2 cells. (A) Cell proliferation in Hep-2 cells was measured using the MTT method as described in Materials and methods. Uninfected Hep-2 cells (Con) and cells infected with the Lv-shNLK and Lv-shCon were seeded in 6-well plates. (B) Colony formation assay. The number of colonies of Hep-2 cells following seeding for 14 days was counted. (C) Colonies stained with Giemsa were observed under fluorescence microscopy (magnification, x200). (D) Hep-2 cells were seeded in 6 well plates at a density of 200 per well and were allowed to form colonies for 14 days. NLK, nemo-like kinase; OD, optical density.

Suppression of cell proliferation and tumorigenesis in Hep-2 cells by si-NLK. To elucidate the role of NLK in cellular proliferation, the proliferative property of Lv-shNLK was analyzed using an MTT assay. The cell proliferation assay was performed for 5 days. The data revealed that there was a significant reduction in Hep-2 cell proliferation as a result of NLK knockdown compared with the uninfected cells (con) and Lv-shCon (Fig. 2A). Notably, 4 and 5 days post-infection, si-NLK-infected cells demonstrated a significantly lower cell viability of $0.58 \pm 0.00$ and $0.60 \pm 0.01$ fold, respectively, compared with the control group $(\mathrm{P}<0.01)$. These findings suggest that NLK is important in Hep-2 cell proliferation.

The results of the colony formation assay also revealed that downregulation of NLK caused a decrease $(83.34 \%)$ in the number of colonies compared with uninfected cells $(\mathrm{P}<0.01$; Fig. 2B). In addition, as evaluated by Giemsa staining and fluorescence microscopy, Hep-2 cells with NLK suppression exhibited a marked reduction in colony size (Fig. 2C and D), suggesting that the tumorigenesis of Hep-2 cells was impaired following suppression of NLK expression.

NLK knockdown arrests the cell cycle at the S phase in Hep-2 cells. The present study also aimed to examine the effects of NLK knockdown on cell cycle progression. Hep-2 cells were labeled with PI and analyzed by DNA flow cytometry. A representative histogram for the Hep-2 cells is shown in Fig. 3A. NLK knockdown caused an increase in the percentage of cells in the $\mathrm{S}$ phase and a corresponding decrease in the percentage of cells in the $\mathrm{G}_{0} / \mathrm{G}_{1}$ and $\mathrm{G}_{2}-\mathrm{M}$ phases (Fig. 3B). In addition, as depicted in Fig. 3C, the percentage of sub- $\mathrm{G}_{1}$ apoptotic cells of Lv-shNLK were significantly higher compared with Lv-shCon and Con.

$N L K$ regulates the expression of cell-cycle regulatory proteins in Hep-2 cells. In addition to the possible molecular mechanism for cell growth inhibition and cell cycle arrest in Hep-2 cells, pro-apoptotic protein expression was also investigated. The protein expression of cell cycle regulatory proteins, including cyclin B1, cyclin D1, p53 and pS315-p53 were examined (Fig. 4). Protein levels of cyclin D1, whose function is important for the $\mathrm{G}_{1}-\mathrm{S}$ transition, were significantly upregulated in Lv-shNLK. In addition, cyclin B1 expression, which controls $\mathrm{G}_{2}-\mathrm{M}$ transition was also upregulated in $\mathrm{Lv}$-shNLK. These results demonstrated that NLK may be involved in translational modification of cell cycle regulatory proteins in Hep-2 cells. Furthermore, Lv-shNLK markedly phosphorylated p53 (Fig. 4), while uninfected cells and Lv-shCon did not exhibit phosphorylated $\mathrm{p} 53$. These results confirm that LV-shNLK specifically phosphorylates $\mathrm{p} 53$ in vitro.

\section{Discussion}

Treatment indications in cancer of the larynx are often controversial, as there are few comparative studies of the different available therapeutic approaches (14). At present, surgery and radiotherapy are widely used and the choice between these 
A
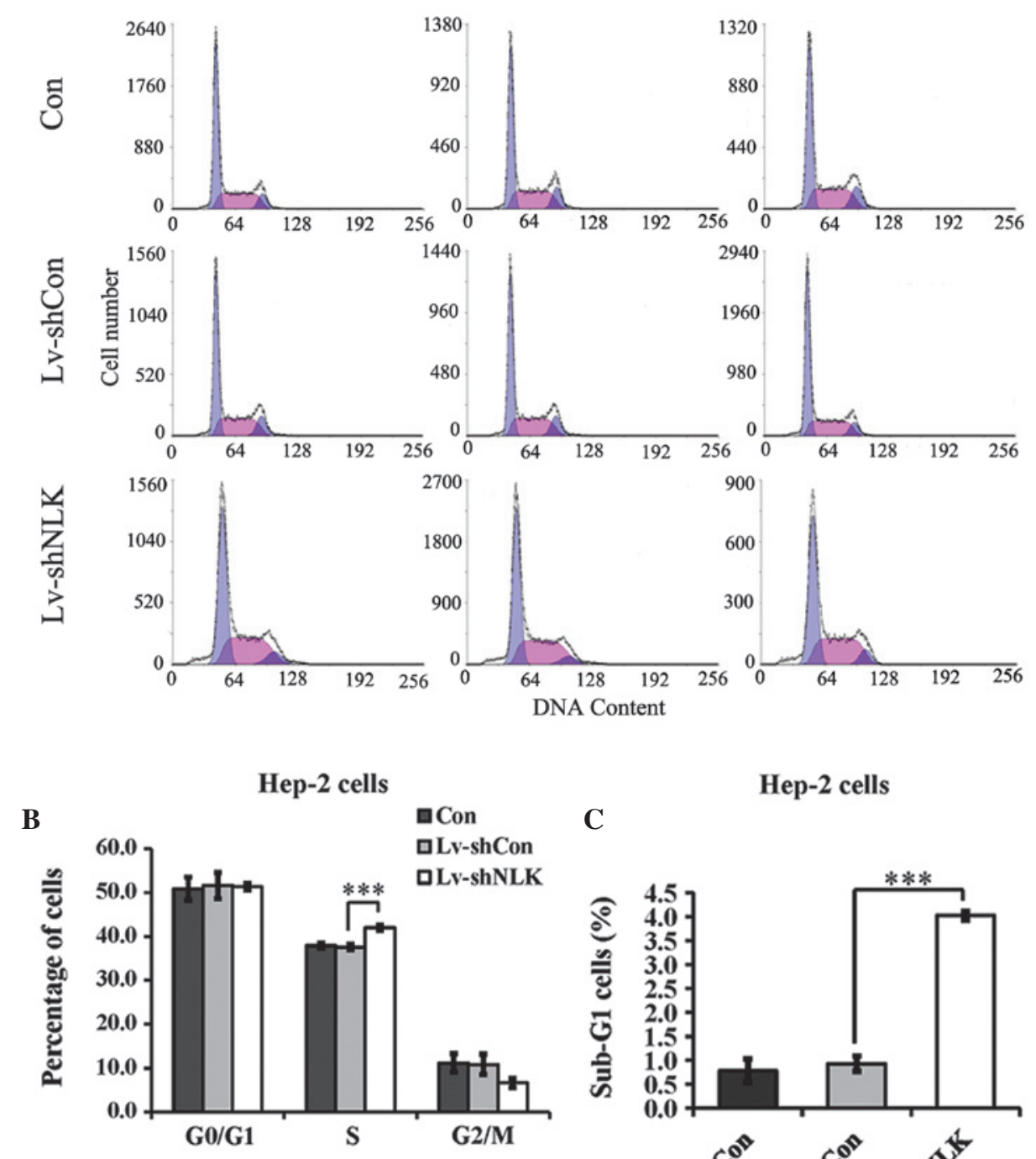

C

Hep-2 cells

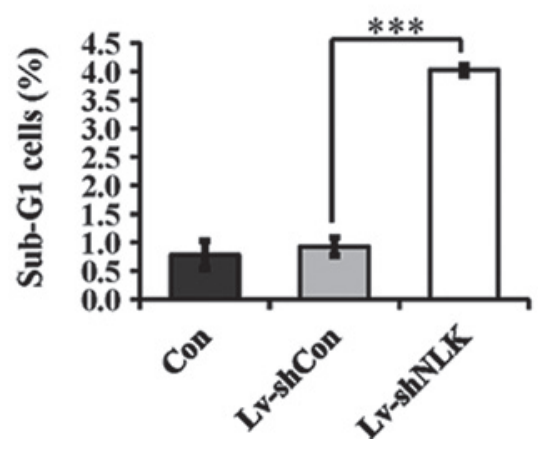

Figure 3. Effects of NLK on the cell cycle and cell apoptosis determined by flow cytometric analysis. (A) Histograms showing the cell cycle distribution of uninfected Hep-2 cells (Con) and cells infected with the Lv-shNLK and Lv-shCon. (B) Suppression of NLK expression by RNA interference resulted in an increase in the percentage of cells in the S phase. (C) A significant increase was identified in the percentage of cells in the sub- $\mathrm{G}_{1}$ phase following transfection with NLK siRNA. The percentage of cells in each phase of the cycle is presented as the mean \pm standard error of three independent experiments. NLK, nemo-like kinase.

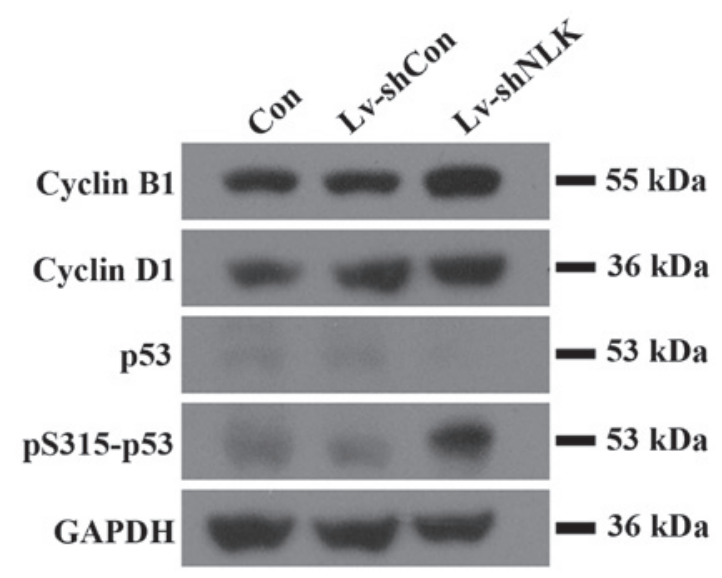

Figure 4. Western blot analysis of apoptosis-associated proteins. Western blot analysis revealed that knockdown of NLK resulted in upregulation of pro-apoptotic proteins in Lv-shNLK cells, suggesting that the apoptotic effect of NLK may be partly mediated by pro-apoptotic family member proteins. two procedures is a common therapeutic decision. Laryngeal function preservation has gained more and more interest in previous decades and chemotherapy is also a significant component of several curative approaches (15). In addition, novel targeted therapeutic methods, including gene therapies are opening a new way to improve the treatment of patients diagnosed with laryngeal cancer.

siRNA is now widely used in cancer studies and may provide a promising method for laryngeal cancer therapy. Use of the lentivirus for gene disruption is popular due to its high efficiency and safety in humans (16). In the present study, the lentivirus technique was employed to specifically inhibit NLK gene expression and demonstrated that loss of NLK function significantly impaired the proliferative and tumorigenic properties of Hep-2 cells. NLK is an MAPK-like kinase, which belongs to the proline-directed serine/threonine protein kinase superfamily. NLK has been revealed to markedly alter 
the activity of other transcription factors, including nuclear factor- $\kappa \mathrm{B}$, Smads, activator protein 1 and p53 (17). The function of NLK in several cancer cell lines has been examined. For example, Jung et al (6) demonstrated that NLK inhibited cell growth and arrested cell cycle transition at the $G_{1} / S$ phase in a Hep3B cell line. By contrast, overexpression of NLK in colon and ovarian cancer cell lines suppressed cell growth and increased programmed cell death $(18,19)$. It has been suggested that these controversial results were at least partially due to the different cancer types investigated, reflecting the complicated function of NLK in the biological progression of different types of cancer (20).

In the present study, NLK downregulation in human laryngeal cancer cells were examined. Suppression of NLK inhibits cell growth and tumorigenesis of Hep-2 cells, as elucidated using MTT, colony formation and flow cytometric analysis. NLK downregulation induces activation of the p53 protein. As a tumor suppressor, p53 is essential for preventing inappropriate cell proliferation, cell cycle arrest, apoptosis, DNA repair in a variety of cell types and maintaining genomic integrity following genotoxic stress (21). The importance of p53 in tumor suppression is highlighted by the observation that approximately half of all types of human cancer exhibit evidence for the loss of normal p53 function due to a mutation within the p53 gene (22). Thus, activation of p53, may partially explain the inhibition of tumorigenesis through NLK silencing. Furthermore, it was found that NLK knockdown reduced the cell survival rate by arresting the cell cycle at $\mathrm{S}$ phase. Progression from the $\mathrm{G}_{1}-\mathrm{S}$ phase of the cell cycle required activation of CDK4, and CDK4 activation is controlled, in part, by a complex formation with its catalytic partner, cyclin D1 (23). The eukaryotic cell cycle is regulated through the sequential activation and inactivation of CDKs, which drive cell cycle progression through the phosphorylation of key regulatory proteins (24). Thus, cyclin D1 is necessary and rate-limiting for $\mathrm{S}$ phase progression. In the present study, the decrease in cell viability may be attributed to the occurrence of $S$ phase cell cycle arrest following knockdown of NLK by RNAi in Hep-2 cells. However, further investigation is required to elucidate the detailed mechanisms.

In conclusion, the present study demonstrated that the RNAi-mediated targeting of NLK regulates Hep-2 cell proliferation. These results also suggest that the siRNA-mediated downregulation of the target gene expression is sufficiently stable in laryngeal cancer cells. In addition, disruption of NLK suppressed tumor cell proliferation. The current data suggested that NLK may be associated with the development of aggressiveness in Hep-2 cells. Furthermore, an improved understanding of NLK function and processing may provide novel insights into the clinical therapy of laryngeal cancer.

\section{References}

1. Wang F, Song G, Liu M, Li X and Tang H: miRNA-1 targets fibronectin 1 and suppresses the migration and invasion of the HEp2 laryngeal squamous carcinoma cell line. FEBS Lett 585: 3263-3269, 2011.
2. Liu T, Zhang M, Zhang $\mathrm{H}$, Sun $\mathrm{C}$ and Deng $\mathrm{Y}$ : Inhibitory effects of cucurbitacin B on laryngeal squamous cell carcinoma. Eur Arch Otorhinolaryngol 265: 1225-1232, 2008.

3. Bezerra de Souza DL, Jerez Roig J and Bernal MM: Laryngeal cancer survival in Zaragoza (Spain): a population-based study. Clin Transl Oncol 14: 221-224, 2012.

4. Dechaphunkul T: Epidemiology, risk factors, and overall survival rate of laryngeal cancer in Songklanagarind Hospital. J Med Assoc Thai 94: 355-360, 2011.

5. Pignon JP, Bourhis J, Domenge $C$ and Designé L: Chemotherapy added to locoregional treatment for head and neck squamous-cell carcinoma: three meta-analyses of updated individual data. Lancet 355: 949-955, 2000.

6. Jung KH, Kim JK, Noh JH, et al: Targeted disruption of Nemo-like kinase inhibits tumor cell growth by simultaneous suppression of cyclin D1 and CDK2 in human hepatocellular carcinoma. J Cell Biochem 110: 687-696, 2010.

7. Meneghini MD, Ishitani T, Carter JC, et al: MAP kinase and Wnt pathways converge to downregulate an HMG-domain repressor in Caenorhabditis elegans. Nature 399: 793-797, 1999.

8. Verheyen EM, Mirkovic I, MacLean SJ, Langmann C, Andrews BC and MacKinnon C: The tissue polarity gene nemo carries out multiple roles in patterning during Drosophila development. Mech Dev 101: 119-132, 2001.

9. Yasuda J, Yokoo H, Yamada T, Kitabayashi I, Sekiya T and Ichikawa H: Nemo-like kinase suppresses a wide range of transcription factors, including nuclear factor- $\kappa$ B. Cancer Sci 95: 52-57, 2004.

10. Sampson EM, Haque ZK, Ku MC, et al: Negative regulation of the Wnt-beta-catenin pathway by the transcriptional repressor HBP1. EMBO J 20: 4500-4511, 2001.

11. Ishitani T, Ninomiya-Tsuji J and Matsumoto K: Regulation of lymphoid enhancer factor $1 / \mathrm{T}$-cell factor by mitogen-activated protein kinase-related Nemo-like kinase-dependent phosphorylation in Wnt/beta-catenin signaling. Mol Cell Biol 23: 1379-1389, 2003.

12. Castanotto D and Rossi JJ: The promises and pitfalls of RNA-interference-based therapeutics. Nature 457: 426-433, 2009.

13. Park F: Lentiviral vectors: are they the future of animal transgenesis? Physiol Genomics 31: 159-173, 2007.

14. Licitra L, Bernier J, Grandi C, et al: Cancer of the larynx. Crit Rev Oncol Hematol 47: 65-80, 2003.

15. Marioni G, Marchese-Ragona R, Cartei G, Marchese F and Staffieri A: Current opinion in diagnosis and treatment of laryngeal carcinoma. Cancer Treat Rev 32: 504-515, 2006.

16. Cheng $K$ and Qin B: RNA interference for cancer therapy. In: Pharmaceutical Perspectives of Cancer Therapeutics. Lu Y and Mahato RI (eds). Springer, US, pp399-440, 2009.

17. Emami KH, Brown LG, Pitts TE, Sun X, Vessella RL and Corey E: Nemo-like kinase induces apoptosis and inhibits androgen receptor signaling in prostate cancer cells. Prostate 69: 1481-1492, 2009.

18. Yasuda J, Tsuchiya A, Yamada T, Sakamoto M, Sekiya T and Hirohashi S: Nemo-like kinase induces apoptosis in DLD-1 human colon cancer cells. Biochem Biophys Res Commun 308: 227-233, 2003.

19. Zhang Y, Peng C, Wu G, et al: Expression of NLK and its potential effect in ovarian cancer chemotherapy. Int J Gynecol Cancer 21: 1380-1387, 2011.

20. Tan Z, Li M, Wu W, et al: NLK is a key regulator of proliferation and migration in gallbladder carcinoma cells. Mol Cell Biochem 369: 27-33, 2012.

21. Bai L and Zhu WG: p53: structure, function and therapeutic applications. J Cancer Mol 2: 141-153, 2006.

22. Dey A, Verma CS and Lane DP: Updates on p53: modulation of p53 degradation as a therapeutic approach. Br J Cancer 98: 4-8, 2008.

23. Sherr CJ and Roberts JM: Living with or without cyclins and cyclin-dependent kinases. Genes Dev 18: 2699-2711, 2004.

24. Gorospe M, Liu Y, Xu Q, Chrest FJ and Holbrook NJ: Inhibition of G1 cyclin-dependent kinase activity during growth arrest of human breast carcinoma cells by prostaglandin A2. Mol Cell Biol 16: 762-770, 1996. 